\title{
Vaginal implantation metastasis of endometrial carcinoma: A case report
}

\author{
YUELING WANG $^{1 *}$, JIANG DU $^{1 *}$, SHULAN LV $^{1}$, YANXIA SUI $^{1}$, XUE XUE $^{1}$, CHAO SUN $^{1}$, \\ JUNKAI ZOU ${ }^{1}$, QUNYING MA ${ }^{1}$, GUOXING FU ${ }^{2}$, QING SONG ${ }^{1,2}$ and QILING LI ${ }^{1}$ \\ ${ }^{1}$ Department of Obstetrics and Gynecology, First Affiliated Hospital of Xi'an Jiaotong University, Xi'an, Shaanxi 710061, \\ P.R. China; ${ }^{2}$ Cardiovascular Research Institute, Morehouse School of Medicine, Atlanta, GA 30310, USA
}

Received April 2, 2015; Accepted April 29, 2016

DOI: $10.3892 / \mathrm{ol} .2016 .4656$

\begin{abstract}
Endometrial cancer is the most common malignancy of the female reproductive system. The three common spread patterns of endometrial cancer are local invasion, lymphatic spread and hematogenous spread. Vaginal metastasis occurs by submucosal lymphatic or vascular metastases in $\sim 10 \%$ of patients with clinical stage I disease. Vaginal implantation metastasis of endometrial cancer is extremely rare. Here we present a case of endometrial carcinoma (International Federation of Gynecology and Obstetrics stage IA) spread to the vagina by implantation metastasis as opposed to any of the methods mentioned above. This conclusion was confirmed mainly from pathological examination. This case highlights the occurrence of vaginal implantation metastasis of endometrial carcinoma. Certain changes may be applied during surgery to prevent implantation metastasis in patients with endometrial cancer.
\end{abstract}

\section{Introduction}

Endometrial cancer is the most frequent malignancy of the female reproductive system. Approximately $70 \%$ of cases with endometrial cancer belong to stage I, and only $8 \%$ present distant metastases to other organs including the lung, liver and bones (1). The main treatment for stage I endometrial cancer is surgery, occasionally followed by radiotherapy, chemotherapy and/or hormonal therapy if high risk factors exist. However,

Correspondence to: Dr Qiling Li, Department of Obstetrics and Gynecology, First Affiliated Hospital of Xi'an Jiaotong University, 277 Yanta West Road, Xi'an, Shaanxi 710061, P.R. China

E-mail: liqiling@mail.xjtu.edu.cn

Abbreviations: FIGO, International Federation of Gynecology and Obstetrics; ER, estrogen receptor; PR, progesterone receptor; PET-CT, positron emission tomography-computed tomography; cGy, centigray.

\section{${ }^{*}$ Contributed equally}

Key words: endometrial carcinoma, vaginal bleeding, vaginal implantation metastasis, postmenopausal bleeding, surgery
$75 \%$ of endometrial cancer recurrence occurs within two or three years of the surgery (2). The three most common recurrent patterns of endometrial cancer are local invasion, lymphatic spread and hematogenous spread (3). Vaginal implantation is extremely rare. Here we present the case of a 77-year-old female with recurrent endometrial carcinoma as a possible pattern of vaginal implantation metastasis differing from any of the patterns mentioned above. Written informed consent was obtained from the patient.

\section{Case report}

Patient presentation. A 77-year-old Chinese female, gravid 4, Para 2, who had experienced the menopause 27 years earlier, was admitted to the First Affiliated Hospital of Xi'an Jiaotong University (Xi'an China) with a 5-day history of vaginal bleeding. The patient had a history of hormone replacement therapy following her menopause. Speculum examination revealed that the cervix was enlarged and smooth, and the vagina was smooth. On bimanual examination, the uterus was observed to be of normal size and the bilateral adnexa were free. Ultrasound examination of the pelvis revealed an endometrial thickness of $\sim 6 \mathrm{~mm}$ with a $2-\mathrm{cm}$ mass in the uterine cavity. Fractional endometrial curettage revealed well-differentiated endometrial adenocarcinoma. The patient's human papillomavirus infection status was unknown. Positron emission tomography-computed tomography (PET-CT) of the chest and whole abdomen revealed no metastatic lesions.

Treatment and findings. The patient's general health was extremely good, and her routine laboratory examinations were considered normal for her age. The patient underwent a total abdominal hysterectomy with bilateral salpingo-oophorectomy. Frozen-section pathological examination during the surgery revealed well-differentiated endometrial adenocarcinoma, with the lesion located only in the superficial muscle layer of the corpus. Histopathological examination following surgery revealed grade 1 endometrial adenocarcinoma (Fig. 1) with the tumor measuring $10 \mathrm{~mm}$ in diameter and myometrial invasion of $4 \mathrm{~mm}$ in depth. No cervical invasion and adnexal metastasis were observed. Immunohistochemical staining revealed positivity for estrogen receptor (ER) and progesterone receptor (PR) (Figs. 2 and 3). According to the International Federation of 


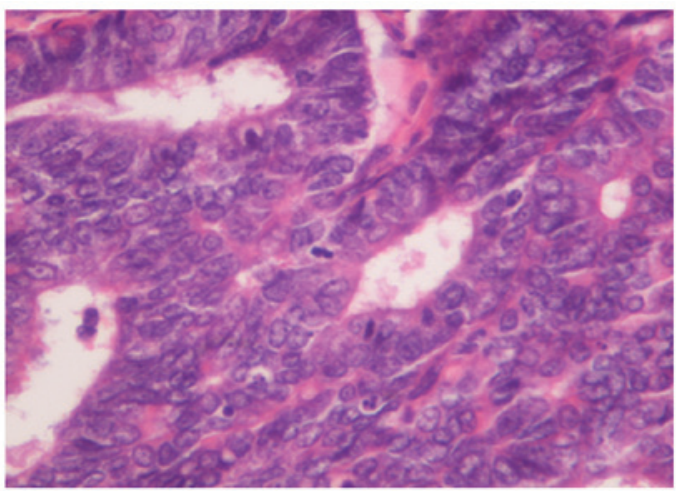

Figure 1. Primary endometrial cancer. A highly-differentiated endometrial adenocarcinoma tumor, measuring $10 \mathrm{~mm}$ in diameter, was identified, with myometrial invasion. Hematoxylin and eosin staining; magnification, x100.

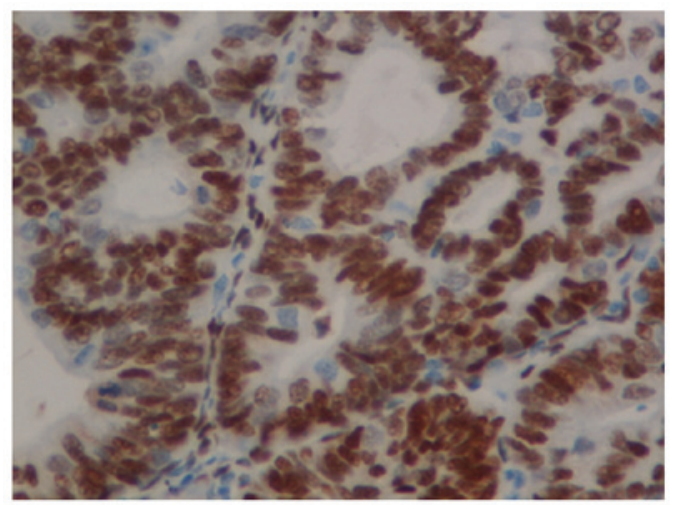

Figure 2. Positive expression of estrogen receptor (ER) in primary endometrial carcinoma. Immunohistochemical staining for ER revealed nuclear positivity restricted to the glands. DAB and hematoxylin and eosin staining; original magnification, $\mathrm{x} 400$.

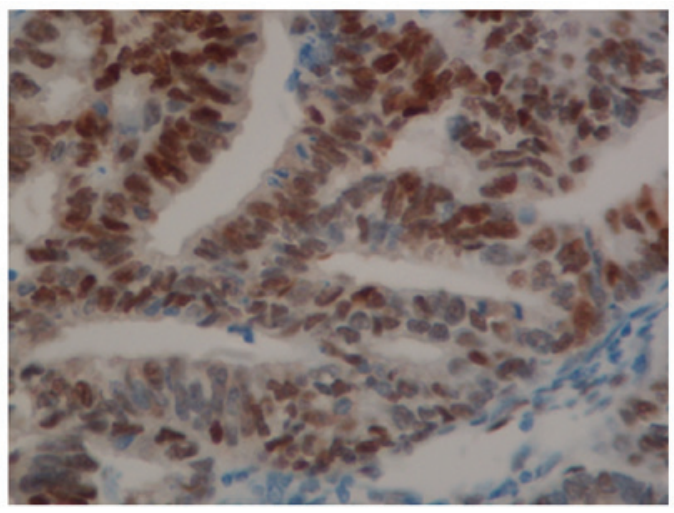

Figure 3. Positive expression of progesterone receptor (PR) in primary endometrial carcinoma. Nuclear staining for PR in the stroma and glands was evident. DAB and hematoxylin and eosin staining; original magnification, x400.

Gynecology and Obstetrics (FIGO) staging system of 2009, the endometrial neoplasm was assessed as stage IA. The patient did not undergo any adjuvant treatment following surgery.

Follow-up treatment and final diagnosis. Half a year after the initial surgery, the patient experienced occasional bloody

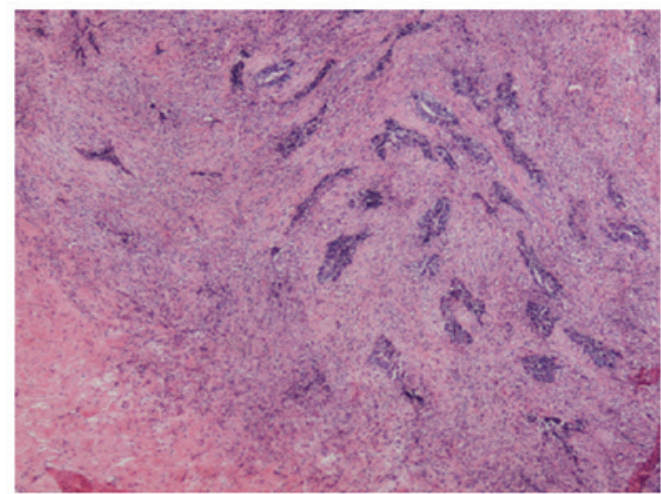

Figure 4. Vaginal implantation metastasis of endometrial cancer. Vaginal mucosa was damaged and the surface squamous epithelium had disappeared. Carcinoma cells were funicular and glandular in the vagina mucosa lamina propria infiltration, and carcinoma tissue fibrosis with inflammatory cell infiltration was observed. Hematoxylin and eosin staining; magnification, x100.

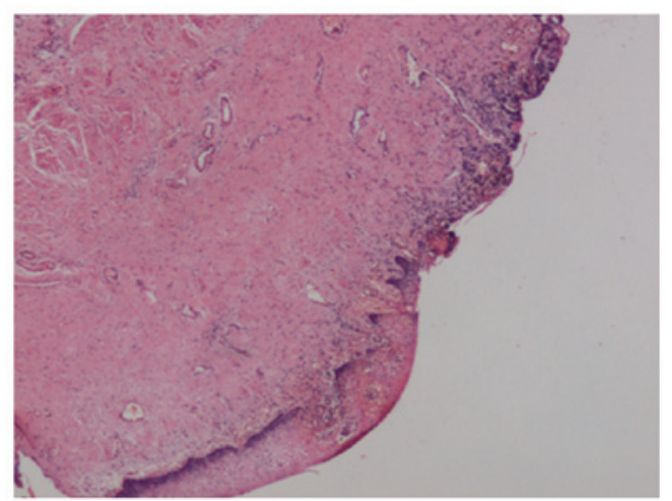

Figure 5. Vaginal tissue was removed. No tumor cells were observed under microscopy. Superficial necrosis was noted adjacent to the normal squamous epithelium. Hematoxylin and eosin staining; magnification, $\mathrm{x} 40$.

vaginal discharge and had vaginal bleeding for two weeks before her admission. Gynecological examination revealed a cauliflower-like tumor mass of $15 \times 15 \times 10 \mathrm{~mm}$ at the eight o'clock position on the upper third of the vaginal wall. PET-CT of the pelvic examination revealed an isolated vaginal mass. There was some loose tissue detaching during the examination, and histopathological study of the loose section revealed well-differentiated endometrial adenocarcinoma. The vaginal mass was removed under general anesthesia. Under a microscope following hematoxylin and eosin staining, the vaginal mucosa was observed to be damaged, and the superficial squamous epithelium had disappeared. The carcinoma cells appeared funicular and glandular in the vaginal mucosa lamina propria infiltration (Fig. 4), and carcinoma tissue fibrosis with inflammatory cell infiltration was observed (Fig. 5). From this microscopic examination, we were able to confirm that the tumor was superficial and localized implantation metastasis. Immunohistochemical staining revealed that ER and PR were positive in the vaginal tumor tissue (Figs. 6 and 7). Following treatment, the patient's vaginal bleeding subsided. The patient had regular follow-ups in the next year. On the recurrence of endometrial carcinoma, the patient received an external pelvic radiotherapy (2 Gray/day, days 1-5) and chemotherapy with 


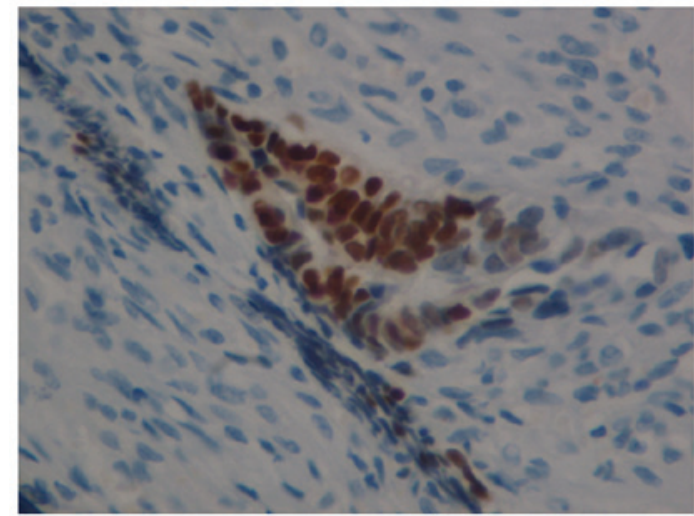

Figure 6. Positive expression of estrogen receptor (ER) in the vaginal tissue. Immunohistochemical study for ER revealed positive nuclear staining. DAB and hematoxylin and eosin staining; magnification, $\mathrm{x} 400$.

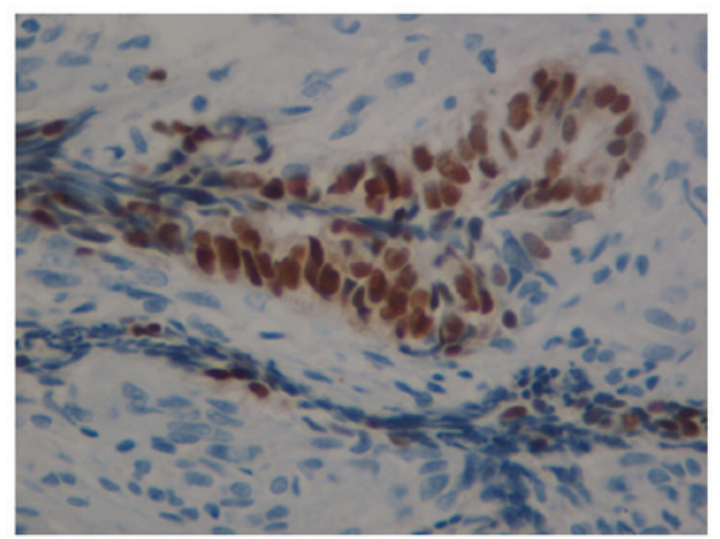

Figure 7. Positive expression of progesterone receptor (PR) in the vaginal tissue. Nuclear immunohistochemical staining for PR in the stroma and glands was positive. DAB and hematoxylin and eosin staining; magnification, $\mathrm{x} 400$.

liposome $\left(135 \mathrm{mg} / \mathrm{m}^{2}\right)$ and carboplatin $\left(300 \mathrm{mg} / \mathrm{m}^{2}\right)$ following the surgery. Following that, the patient received vaginal brachytherapy with four applications of 500 centigray (cGy) (total dose of 2000 cGy). During the two-year follow-up after treatment, there was no any sign of recurrence.

\section{Discussion}

We deduce that the recurrence of vaginal tumors may be considered as a type of implantation metastasis. This conclusion is mainly deduced from pathological examination. It is known that $90 \%$ of endometrial cancers are adenocarcinomas, and that most vaginal cancers are squamous cell carcinomas (4). In the present case, the vaginal tumor was histologically identical to primary endometrial adenocarcinoma. The expression of ER and PR was positive in the vaginal tumor as well as in the endometrial carcinoma. The vaginal mucosa was damaged and the vaginal squamous epithelium had dissolved. The carcinoma cells are a funicular and glandular inside the vaginal mucosa lamina propria infiltration, and carcinoma tissue fibrosis with inflammatory cell infiltration. Histopathological study revealed a superficial and localized implantation metastasis tumor. Moreover, there was no sign of lymphatic or hematogenous spread of the primary endometrial carcinoma. Therefore, the vaginal mass could be considered as an implantation metastasis tumor of the primary endometrial carcinoma.

There is no clear cause of vaginal implantation metastasis, and how to minimize this implantation remains unclear. Here, we present several possible mechanisms of vaginal implantation metastases of endometrial cancer. First of all, the possible spread pattern may be attributed to iatrogenic transtubal spill (3). Endometrial adenocarcinoma cell dissemination during hysteroscopy and saline infusion sonography may increase the risk of spill. The second possibility is that high-grade endometrial cancers have implantation capacity, and as a result, peritoneal invasion (5) or vaginal implantation occurs. The shed tissue during the examination or surgery of patients with endometrial cancer may cause endometrial cancer cells to implant in a suitable location. These cells could survive and be capable of invasion and metastasis (6).

To avoid implantation metastasis of endometrial carcinoma in these patients, certain changes may be made in the surgical procedure. First of all, when surgery for endometrial cancers is performed, the initial surgical site should be examined carefully (7), and the surrounding tissue should be carefully protected to avoid implantation of the tumor cells. Secondly, cervical cerclage, ligation of the fallopian tubes and vaginal douching should be performed during surgery to prevent carcinoma cells from implanting in other organs. Thirdly, reducing unnecessary gynecological examinations is expected to lower the possibility of cancer cell dissemination. Further research should be carried out to establish the actual mechanisms of vaginal implantation metastases of endometrial carcinoma.

\section{References}

1. Ma XG, Wang YM, Sheng HN, Qi Z, Tian WY, Liu GY and Xue FX: Endometrial cancer metastasize to the skin of lower leg and vagina: Case report and literature review. Eur J Gynaecol Oncol 34: 350-352, 2013

2. Tejerizo-Garcia A, Álvarez-Conejo C, Muñoz-Hernando L, Guillñn-Gámez C, Seoane-Ruiz JM, Pñrez-Sagaseta C and Jimñnez-López JS: Tumor recurrence and tumor-related mortality in endometrial cancer: Analysis in 276 patients. Indian J Cancer 52: 682-684, 2015.

3. Guralp O and Kushner DM: Iatrogenic transtubal spill of endometrial cancer: risk or myth. Arch Gynecol Obstet 284: 1209-1221, 2011.

4. Woelber L1, Kock L, Gieseking F, Petersen C, Trillsch F, Choschzick M, Jaenicke F, Mahner S: Clinical management of primary vulvar cancer. Eur J Cancer 47: 2315-2321, 2011.

5. Stewart CJ, Doherty DA, Havlat M, Koay MH, Leung YC, Naran A, O'Brien D, Ruba S, Salfinger S and Tan J: Transtubal spread of endometrial carcinoma: Correlation of intra-luminal tumour cells with tumour grade, peritoneal fluid cytology, and extra-uterine metastasis. Pathology 45: 382-387, 2013.

6. Atallah D, el Kassis N, Lutfallah F, Safi J, Salameh C, Nadiri S and Bejjani L: Cutaneous metastasis in endometrial cancer: once in a blue moon - case report. World J Surg Oncol 12: 86, 2014.

7. Nguyen ML, Friedman J, Pradhan TS, Pua TL and Tedjarati SS: Abdominal wall port site metastasis after robotically staged endometrial carcinoma: A case report. Int J Surg Case Rep 4: 613-615, 2013. 